EDITORIAL

\title{
Integrative cancer genomics in the era of precision cancer medicine
}

(c) The Author(s), under exclusive licence to The Japan Society of Human Genetics 2021

Journal of Human Genetics (2021) 66:843; https://doi.org/10.1038/s10038021-00970-6

Cancer is a disease caused by genomic and epigenome abnormalities, and the accumulation of such abnormalities and errors results in cancer evolution. The development of nextgeneration sequencers (NGSs) led to a large-scale cancer genome sequence project, which revealed the existence of a huge number of nucleic acid sequence alterations in the genomes of various cancer cells.

Among them, the mutation that contributes to actual carcinogenesis is called a cancer driver mutation. When a protein product translated by a mutated gene has the ability of oncogenesis, a specific inhibitor for that product has a remarkable cancer inhibitory effect, that is, molecular target therapy. Many genetic abnormalities responsible for such carcinogenesis have been identified in various cancers. Molecular target therapies have demonstrated remarkable clinical success in the treatment of various cancers including breast, colorectal, lung, gastric, liver, and gynecologic cancers. Currently, with the aim of selecting effective therapeutic drugs, genetic diagnosis of cancer not only uses a system that comprehensively diagnoses many genetic mutations simultaneously with tissues obtained by surgical resection, a socalled cancer gene panel, but also a liquid biopsy method that reduces invasion of patients using DNA leaking from cancer cells and cancer cells circulating in the patient's peripheral blood.

NGS has also revealed the presence of age-related somatic mutations in the human genome, which are important for understanding the background of different cancer developments among environments, races, and countries. Immunogenomics has developed in the field of clinical implementations of immunecheckpoint inhibitors, CAR-T-cell therapy and cancer vaccines.
Increasing evidence on the functional roles of noncoding miRNAs in cancer progression has revealed the potential of miRNA therapeutics in cancer. Furthermore, recent advances in singlecell sequencing enables an omics approach at the single-cell level to reveal the complex interplay between genotype and phenotype. Elucidation of intra-tumor heterogeneity by single-cell DNA sequencing and multi-omics will greatly contribute to the research leading to precision medicine, such as elucidation of the mechanism of cancer recurrence and drug resistance, and development of new methods of cancer therapy.

In the era of "Precision Cancer Medicine," where "whole genome analysis" and "whole exome analysis," that comprehensively analyze an individual's genome sequence (personal genome), are widely used to elucidate the causes of cancers as the monogenic or multifactorial diseases, it is important for us to understand ethical issues such as privacy issues, the management of secondary findings, and challenges in data storage and sharing.

In this special issue of Cancer Genomics, we invite established leaders in cancer omics and/or integrative cancer genomics, most of them are active researchers to discuss about cancer genomics studies and their future perspectives. We hope that this collection of review articles contributes to equip the readers for the era of precision cancer medicine.

Johji Inazawa ${ }^{1,2}$, Yoshio Miki ${ }^{3}$ and Yusuke Nakamura ${ }^{4}$ ${ }^{1}$ Department of Molecular Cytogenetics, Medical Research Institute, Tokyo Medical and Deantal University, Tokyo, Japan. ${ }^{2}$ Bioresource Research Center, Tokyo Medical and Dental University, Tokyo, Japan.

${ }^{3}$ Department of Molecular Genetics, Medical Research Institute, Tokyo Medical and Dental University, Tokyo, Japan. ${ }^{4}$ Cancer Precision Medicine Center, Japanese Foundation for Cancer Research, Tokyo, Japan. ${ }^{\varpi}$ email: johinaz.cgen@mri.tmd.ac.jp 